\title{
Prognostic impact of stress echocardiography with discordant stress electrocardiography in patients with suspected coronary artery disease
}

\author{
Siang Chew $\underline{\text { Chai }}{ }^{1}$, MBBS, MRCP, Hooi Khee $\underline{T e 0^{1}}$, MBBS, MRCP, Pei Shan Lee ${ }^{2}$, MBBS, MrCP, Carmen Jia Wen $\underline{K a m}^{3}$, MSc,
} Khim Leng Tong $^{1}$, MBBS, FRCP

\begin{abstract}
INTRODUCTION During stress echocardiography, the echocardiologist routinely collects both echocardiographic images and stress electrocardiogram (ECG) concurrently. The managing physician faces a dilemma when the stress ECG and stress echocardiography results are discordant; for example, when a patient has negative stress echocardiography but positive stress ECG. We therefore sought to evaluate the prognostic value of stress echocardiography in relation to concordant or discordant stress ECG findings in our local Singapore setting, which has a well-defined Southeast Asian population. METHODS This was a retrospective observational study of all patients who underwent stress echocardiography in 2012 at Changi General Hospital, Singapore. All study patients were followed up for 18 months via electronic medical records. RESULTS There was no difference in the major adverse cardiovascular events (MACE) outcome of patients with normal stress echocardiography and normal stress ECG (reference group) as compared with patients with normal stress echocardiography but positive (discordant) stress ECG (odds ratio 2.02, 95\% confidence interval 0.82-4.98; $p=0.125$ ). CONCLUSION This study will help to reassure cardiologists that discordant results (negative stress echocardiography but positive stress ECG) do not portend a higher risk of MACE when compared to concordant results (i.e. both stress echocardiography and stress ECG are negative).
\end{abstract}

Keywords: discordantstress electrocardiography, prognostic value ofstress echocardiography and stress electrocardiography, stress echocardiography, stress electrocardiography

\section{INTRODUCTION}

Positive stress echocardiography is associated with a threefold increased incidence of a cardiac event and a fourfold increased incidence of myocardial infarction within 12 months of follow-up when compared with negative stress echocardiography. ${ }^{(1)}$ During stress echocardiography, the echocardiologist routinely collects both echocardiographic images and stress electrocardiogram (ECG) concurrently. The managing physician faces a dilemma when the stress ECG and stress echocardiography results are discordant (e.g. when a patient has negative stress echocardiography but positive stress ECG). A positive stress ECG could indicate a high risk of imminent cardiac event, whereas negative stress echocardiography would indicate the contrary. ${ }^{(2)}$ However, Kobal et $\mathrm{a}^{(3)}$ and Mahenthiran et $\mathrm{al}^{(4)}$ have demonstrated, in their studies, that a normal finding on stress echocardiography confers a benign prognosis independent of the type of stress ECG response during stress studies. ${ }^{(3,4)}$ We therefore sought to evaluate the prognostic value of stress echocardiography in relation to concordant or discordant stress ECG findings in our local Singapore setting, which has a well-defined Southeast Asian multi-ethnic population.

\section{METHODS}

We conducted a retrospective observational study of all patients who underwent stress echocardiography in 2012 at Changi General Hospital (CGH), Singapore. CGH is a 1,000-bed tertiary hospital in eastern Singapore, serving a population of 1.6 million people. The study was approved by the local institutional review board. All study patients were followed up for 18 months via electronic medical records using the Singapore National Electronic Health Record system.

A resting echocardiogram was performed in the lateral decubitus position. Resting digital images, with tissue harmonic imaging of the left ventricle, were obtained in the parasternal long-axis, short-axis, and apical four- and two-chamber views using the iE 33 xMATRIX echocardiography system (Koninklijke Philips, Eindhoven, Netherlands) or Vivid E9 echocardiography system (GE Healthcare, Chicago, IL, USA).

Exercise stress echocardiography images were acquired immediately (within 90 seconds) after peak exercise. Immediate post-exercise images, with the best endocardial definition, were selected and displayed alongside the corresponding baseline images. Exercise testing was symptom-limited and was stopped for standard criteria as follows: moderate-to-severe chest pain; fatigue; shortness of breath; and significant arrhythmia. The patients were expected to achieve a target heart rate defined as $>85 \%$ maximal age-predicted heart rate. Failure to achieve the target heart rate was defined as submaximal exercise testing, and these patients were not to be included in the data analysis.

For patients who were not able to perform the treadmill exercise, pharmacologic stress testing with dobutamine infusion was administered instead, according to American Society of Echocardiography 2007 guidelines. ${ }^{(5)}$ A graded dobutamine infusion starting at $5 \mu \mathrm{g} / \mathrm{kg} /$ minute and increasing at three-minute

${ }^{1}$ Department of Cardiology, ${ }^{2}$ Department of Renal Medicine, ${ }^{3} \mathrm{Clinical}$ Trials and Research Unit, Changi General Hospital, Singapore

Correspondence: Dr Chai Siang Chew, Consultant, Department of Cardiology, Changi General Hospital, 2 Simei Street 3, Singapore 529889. chai.siang.chew@singhealth.com.sg 




Fig. 1 Flow diagram shows patient recruitment into the study. ECG: electrocardiogram; ECG-: negative stress electrocardiogram; ECG+: positive stress electrocardiogram; Echo-: negative stress echocardiography; Echo+: positive stress echocardiography

intervals to $10 \mu \mathrm{g} / \mathrm{kg} /$ minute, $20 \mu \mathrm{g} / \mathrm{kg} /$ minute, $30 \mu \mathrm{g} / \mathrm{kg} /$ minute and $40 \mu \mathrm{g} / \mathrm{kg} / \mathrm{minute}$ was the standard for dobutamine stress testing. Atropine in divided doses of $0.3 \mathrm{mg}$ to a total of $2.4 \mathrm{mg}$ would be used, as needed, to achieve the target heart rate. Endpoints were achievement of target heart rate, new or worsening wall motion abnormalities of moderate degree, significant arrhythmias, hypotension, severe hypertension, and intolerable symptoms. Function in each segment was graded at rest and with stress as normal or hyperdynamic, hypokinetic, akinetic, dyskinetic or aneurysmal. Images from low or intermediate stages of dobutamine infusion were compared with peak stress images to maximise the sensitivity for detection of coronary disease.

In technically difficult patients, with two or more left ventricular segments not adequately visualised at rest or during deep breathing, intravenous contrast (Sonovue; Bracco, Milan, Italy) was used to enhance endocardial border definition. Bolus injections of $0.3-0.5 \mathrm{~mL}$ were administered through a peripheral cannula followed by a slow flush with normal saline solution.

Online digital images were interpreted qualitatively for the presence, extent and location of regional wall motion abnormalities by the performing echocardiologist as per routine clinical practice. A normal stress echocardiogram result was defined as normal left ventricle wall motion at rest and with stress. Resting wall motion abnormalities, unchanged with stress, were classified as 'fixed' and most often represented regions of prior infarction. This group would still be classified as negative for inducible ischaemia. Only abnormal study findings for those with new or worsening wall motion abnormalities were classified as positive for inducible ischaemia. Patients with uninterpretable images or patients who failed to achieve the target heart rate were considered to have an inconclusive test.

Stress electrocardiography was performed concurrently during stress echocardiography by experienced cardiac physiologists. Tests were reported as negative, positive, borderline or inconclusive for ischaemia according to predetermined parameters by the performing echocardiologist. Stress ECG results were defined as positive (ischaemic) if there was $\geq 1 \mathrm{~mm}$ ST-segment depression 0.08 seconds after the J point, and it was present in at least three consecutive beats in at least two contiguous leads during peak stress.

Demographics and clinical characteristics of all patients who underwent stress echocardiography were compared using one-way analysis of variance (ANOVA) for numerical variables, and chi-square test or Fisher's exact test for categorical variables, wherever appropriate. If statistically significant differences were found in ANOVA, Bonferroni post-hoc adjustment was used for multiple pairwise comparisons. Outcomes at 18 months were analysed using a logistic regression model, including the following potential confounding covariates: age; gender; ethnicity; previous history of ischaemic heart disease; previous coronary artery bypass grafting (CABG); diabetes mellitus; hypertension; and hyperlipidaemia; and usage of medications, including aspirin, clopidogrel and $\beta$-blockers, angiotensin converting enzyme inhibitor (ACEI), or angiotensin II receptor blocker (ARB).

The endpoints analysed were all-cause mortality and major adverse cardiovascular events (MACE), defined as non-fatal myocardial infarction and coronary revascularisation, including percutaneous coronary intervention ( $\mathrm{PCl}$ ) and $\mathrm{CABG}$. Two-tailed $\mathrm{p}<0.05$ was considered to be statistically significant. Statistical analysis was performed using IBM SPSS Statistics version 19.0 (IBM Corp, Armonk, NY, USA).

\section{RESULTS}

We performed stress echocardiography for a total of 914 patients in 2012. Of these, 13 patients were excluded from analysis due to non-interpretable stress ECG findings, inconclusive stress echocardiography results due to submaximal target heart rate 
Table I. Baseline characteristics of patients who underwent both stress echocardiography and stress electrocardiography.

\begin{tabular}{|c|c|c|c|c|c|}
\hline \multirow[t]{2}{*}{ Variable } & \multicolumn{4}{|c|}{ No. (\%) } & \multirow[t]{2}{*}{ p-value } \\
\hline & $\begin{array}{l}\text { Group A (both } \\
\text { negative) }(n=671)\end{array}$ & $\begin{array}{l}\text { Group B (Echo+ and } \\
\text { ECG-) }(n=47)\end{array}$ & $\begin{array}{l}\text { Group C (Echo- and } \\
\text { ECG }+)(n=122)\end{array}$ & $\begin{array}{l}\text { Group D (both } \\
\text { positive) }(n=61)\end{array}$ & \\
\hline Age $^{*}(y r)$ & $59.4 \pm 14.0$ & $66.1 \pm 11.1$ & $59.4 \pm 12.4$ & $64.5 \pm 11.6$ & $<0.001^{+}$ \\
\hline Male gender & $394(58.7)$ & $29(61.7)$ & $72(59.0)$ & $51(83.6)$ & 0.002 \\
\hline Ethnicity & & & & & 0.026 \\
\hline Chinese & $479(71.4)$ & $31(66.0)$ & $96(78.7)$ & $36(59.0)$ & \\
\hline Malay & $114(17.0)$ & $14(29.8)$ & $15(12.3)$ & $16(26.2)$ & \\
\hline Other & $78(11.6)$ & $2(4.3)$ & $11(9.0)$ & $9(14.8)$ & \\
\hline No. of comorbidities ${ }^{\ddagger}$ & & & & & $<0.001$ \\
\hline 0 & $231(34.4)$ & $4(8.5)$ & $42(34.4)$ & $4(6.6)$ & \\
\hline 1 & $132(19.7)$ & $5(10.6)$ & $29(23.8)$ & $14(23.0)$ & \\
\hline$>1$ & $308(45.9)$ & $38(80.9)$ & $51(41.8)$ & $43(70.5)$ & \\
\hline Previous history of IHD and/or CABG & $127(18.9)$ & $26(55.3)$ & $20(16.4)$ & $35(57.4)$ & $<0.001$ \\
\hline Plavix & $61(9.1)$ & $17(36.2)$ & $10(8.2)$ & $12(19.7)$ & $<0.001$ \\
\hline Aspirin & $168(25.0)$ & $30(63.8)$ & $29(23.8)$ & $46(75.4)$ & $<0.001$ \\
\hline ACEI/ARB and/or $\beta$-blocker & $304(45.3)$ & $38(80.9)$ & $52(42.6)$ & $44(72.1)$ & $<0.001$ \\
\hline
\end{tabular}

*Data presented as mean \pm standard deviation. + Bonferroni post-hoc test showed that significance with respect to age existed for Group B and Group D, which had older patients than Group A ( $p=0.006$ and $p=0.029$, respectively). Group B was also significantly older than Group C $(p=0.023)$. Other comparisons were not statistically significant. łIncluded hypertension, hyperlipidaemia and diabetes mellitus. ACEl: angiotensin-converting enzyme inhibitor; ARB: angiotensin II receptor blocker; CABG: coronary artery bypass grafting; ECG-: negative stress electrocardiogram; ECG+: positive stress electrocardiogram; Echo-: negative stress echocardiography; Echo+: positive stress echocardiography; IHD: ischaemic heart disease

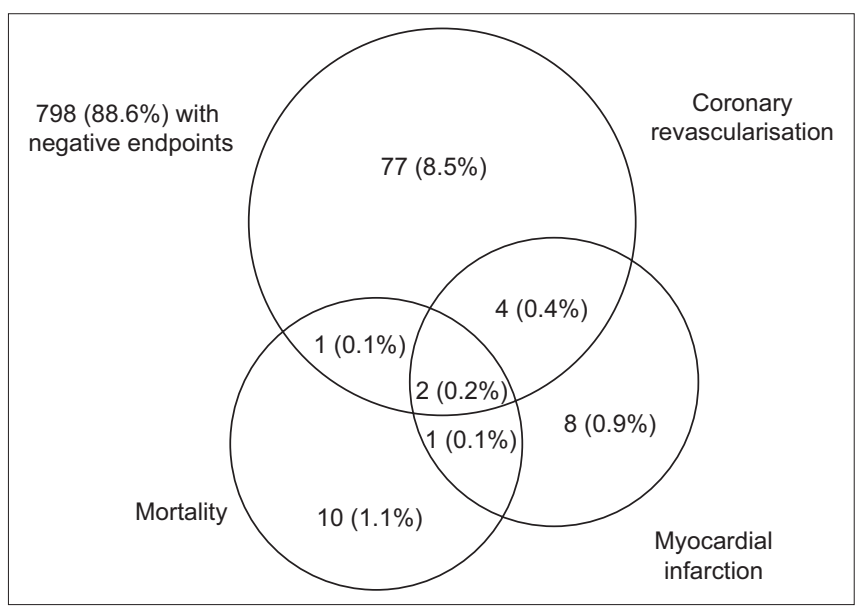

Fig. 2 Diagram shows prevalence of mortality, myocardial infarction and coronary revascularisation among recruited patients.

achieved or incomplete clinical patient information. Fig. 1 shows a flow diagram of patient recruitment into the study.

Primary outcome measurements were MACE (including all-cause and cardiovascular mortality), non-fatal myocardial infarction and coronary revascularisation (including $\mathrm{PCl}$ and CABG). Secondary outcomes included either all-cause mortality and/or coronary revascularisation defined as $\mathrm{PCl}$ or $\mathrm{CABG}$ surgery. Fig. 2 shows the incidence of mortality, myocardial infarction and coronary revascularisation among recruited patients. Overall, 798 (88.6\%) of 901 patients had negative endpoints.

Baseline characteristics for the four patient groups A-D are listed in Table I. Patients with abnormal stress echocardiography were older, less likely to be female and more likely to have more than one comorbidity (e.g. hypertension, hyperlipidaemia or diabetes mellitus). A larger number of patients had a prior history of ischaemic heart disease or CABG, and were already on aspirin, clopidogrel and either ACEI, ARB or $\beta$-blocker.

Table II presents the findings of logistic regression analyses, with adjustment for baseline demographics and comorbidities. The multivariate predictors for primary outcome (i.e. MACE) were positive stress echocardiography (Echo+ and ECG+ [Group D]: odds ratio [OR] 20.57, p < 0.001; Echo+ and ECG- [Group B]: OR 20.31, p < 0.001), presence of more than one risk factor comprising hypertension, hyperlipidaemia and diabetes mellitus (OR 2.93, $\mathrm{p}=0.046$ ), and prior usage of aspirin (OR 1.92, $\mathrm{p}=0.041$ ).

There was no difference in MACE outcomes (Table III) for patients in Group A with normal stress echocardiogram and normal stress ECG when compared to patients in Group C with normal stress echocardiogram but positive (discordant) stress ECG (OR 2.02, 95\% confidence interval [Cl] 0.82-4.98; $p=0.125$ ).

Among 122 patients in Group $\mathrm{C}$ with normal stress echocardiography but positive (discordant) stress ECG, nine patients experienced a primary outcome during the 18 months of follow-up. Two of these patients died - one due to colorectal cancer and the other due to pneumonia. Among patients in Group C, 13 (10.7\%) patients underwent diagnostic coronary angiogram at the managing physicians' discretion - 7 (5.7\%) patients had minor coronary artery disease or ischaemic heart disease for medical treatment, and 6 (4.9\%) patients required $\mathrm{PCl}$. One patient underwent computerised tomography coronary angiogram instead, which showed minor coronary disease. One patient had acute ST elevation myocardial infarction four months after stress echocardiography.

$60(55.6 \%)$ of 108 patients with positive stress echocardiography had coronary revascularisation. Positive stress echocardiography 
Table II. Univariate and multivariate predictors for all-cause mortality and/or combined cardiovascular events.

\begin{tabular}{|c|c|c|c|c|c|c|}
\hline \multirow[t]{2}{*}{ Variable } & \multicolumn{2}{|c|}{ No. (\%) } & \multicolumn{2}{|c|}{ Univariate analysis } & \multicolumn{2}{|c|}{ Multivariate analysis } \\
\hline & No $(n=798)$ & Yes $(n=103)$ & OR $(95 \% \mathrm{Cl})$ & p-value & OR $(95 \% \mathrm{Cl})$ & p-value \\
\hline \multicolumn{7}{|c|}{ Stress echocardiogram and exercise stress ECG } \\
\hline Group A (both negative; $\mathrm{n}=671$ ) & $643(95.8)$ & $28(4.2)$ & 1.00 & & 1.00 & \\
\hline Group B (Echo+ and ECG $-; n=47)$ & $18(38.3)$ & $29(61.7)$ & $37.03(18.46-74.59)$ & $<0.001$ & $20.31(9.50-43.38)$ & $<0.001^{+}$ \\
\hline Group C (Echo- and ECG+; $\mathrm{n}=122)$ & $113(92.6)$ & $9(7.4)$ & $1.83(0.84-3.98)$ & 0.128 & $2.18(0.97-4.91)$ & 0.061 \\
\hline Group $\mathrm{D}$ (both positive; $\mathrm{n}=61$ ) & $24(39.3)$ & $37(60.7)$ & $35.48(18.71-67.09)$ & $<0.001$ & $20.57(10.01-41.94)$ & $<0.001^{+}$ \\
\hline \multicolumn{7}{|l|}{ Age group (yr) } \\
\hline$<50(n=194)$ & $186(95.9)$ & $8(4.1)$ & 1.00 & & 1.00 & \\
\hline $50-59(n=245)$ & $212(86.5)$ & $33(13.5)$ & $3.62(1.63-8.03)$ & 0.002 & $1.97(0.72-5.40)$ & 0.188 \\
\hline $60-69(n=220)$ & $192(87.3)$ & $28(12.7)$ & $3.39(1.51-7.63)$ & 0.003 & $1.51(0.53-4.30)$ & 0.443 \\
\hline $70-79(n=179)$ & $157(87.7)$ & $22(12.3)$ & $3.26(1.41-7.52)$ & 0.006 & $1.33(0.45-3.92)$ & 0.606 \\
\hline$\geq 80(n=63)$ & $51(81.0)$ & $12(19.0)$ & $5.47(2.12-14.15)$ & $<0.001$ & $1.47(0.42-5.14)$ & 0.543 \\
\hline \multicolumn{7}{|l|}{ Gender } \\
\hline Female $(n=355)$ & $328(92.4)$ & $27(7.6)$ & 1.00 & & 1.00 & \\
\hline Male $(n=546)$ & $470(86.1)$ & $76(13.9)$ & $1.96(1.24-3.12)$ & 0.004 & $1.36(0.73-2.53)$ & 0.329 \\
\hline \multicolumn{7}{|l|}{ Ethnicity } \\
\hline Chinese $(n=642)$ & $574(89.4)$ & $68(10.6)$ & 1.00 & & 1.00 & \\
\hline Malay $(n=159)$ & $129(81.1)$ & 30 (18.9) & $1.96(1.23-3.14)$ & 0.005 & $1.54(0.81-2.96)$ & 0.191 \\
\hline Other $(n=100)$ & $95(95.0)$ & $5(5.0)$ & $0.44(0.18-1.13)$ & 0.089 & $0.41(0.13-1.23)$ & 0.111 \\
\hline \multicolumn{7}{|l|}{ No. of comorbidities* } \\
\hline $0(n=281)$ & $275(97.9)$ & $6(2.1)$ & 1.00 & & 1.00 & \\
\hline $1(n=180)$ & $166(92.2)$ & $14(7.8)$ & $3.87(1.46-10.3)$ & 0.007 & $1.64(0.52-5.17)$ & 0.397 \\
\hline$>1(n=440)$ & $357(81.1)$ & $83(18.9)$ & $10.7(4.59-24.8)$ & $<0.001$ & $2.93(1.02-8.45)$ & $0.046^{+}$ \\
\hline \multicolumn{7}{|l|}{ Previous history of IHD and/or CABG } \\
\hline No $(n=693)$ & $651(93.9)$ & $42(6.1)$ & 1.00 & & 1.00 & \\
\hline Yes $(n=208)$ & $147(70.7)$ & $61(29.3)$ & $6.43(4.18-9.91)$ & $<0.001$ & $1.70(0.90-3.21)$ & 0.105 \\
\hline \multicolumn{7}{|l|}{ Clopidogrel } \\
\hline No $(n=800)$ & $727(90.9)$ & $73(9.1)$ & 1.00 & & 1.00 & \\
\hline Yes $(n=100)$ & $70(70.0)$ & $30(30.0)$ & $4.27(2.61-6.97)$ & $<0.001$ & $1.44(0.72-2.89)$ & 0.308 \\
\hline \multicolumn{7}{|l|}{ Aspirin } \\
\hline No $(n=627)$ & $597(95.2)$ & $30(4.8)$ & 1.00 & & 1.00 & \\
\hline Yes $(n=273)$ & $200(73.3)$ & $73(26.7)$ & $7.26(4.61-11.4)$ & $<0.001$ & $1.92(1.03-3.60)$ & $0.041^{+}$ \\
\hline \multicolumn{7}{|l|}{ ACEI/ARB and/or $\beta$-blocker } \\
\hline No $(n=462)$ & $444(96.1)$ & $18(3.9)$ & 1.00 & & 1.00 & \\
\hline Yes $(n=438)$ & $353(80.6)$ & 85 (19.4) & $5.94(3.51-10.11)$ & $<0.001$ & $1.43(0.68-3.00)$ & 0.345 \\
\hline
\end{tabular}

$\mathrm{OR}=1.00$ was reference category. ${ }^{*}$ Included hypertension, hyperlipidaemia and diabetes mellitus. $\dagger \mathrm{p}<0.05$ was statistically significant. ACEl: angiotensin-converting enzyme inhibitor; ARB: angiotensin II receptor blocker; CABG: coronary artery bypass grafting; Cl: confidence interval; ECG: electrocardiogram; ECG-: negative stress electrocardiogram; ECG+: positive stress electrocardiogram; Echo-: negative stress echocardiography; Echo+: positive stress echocardiography; IHD: ischaemic heart disease; OR: odds ratio

(Table III) was the strongest predictor of primary outcome, with OR at 45.39 for patients with negative stress ECG (i.e. Group B; 95\% Cl 22.09-93.28, $\mathrm{p}<0.001$ ); and OR at 35.48 for patients with positive stress ECG (i.e. Group D; 95\% Cl 18.44-68.26, $\mathrm{p}<0.001)$. Of note, positive stress echocardiography and stress ECG (Group D) was the only variable that predicted mortality for the next 18 months (OR 4.54, 95\% Cl 1.18-17.50, $\mathrm{p}=0.028$ ) (Table IV).

\section{DISCUSSION}

To our knowledge, this was one of the few studies that compared the characteristics and clinical outcomes of a well-defined cohort of patients who had undergone stress echocardiography. Data regarding the prognostic significance of abnormal stress echocardiography has been well demonstrated in several studies. ${ }^{(6-12)}$ Our study also consistently demonstrated that patients with positive stress echocardiography, regardless of stress ECG findings, will have a higher risk of developing MACE (OR 20.78, $p<0.001)$. Of note, patients with more than one of the following comorbidities, such as hypertension, hyperlipidaemia or diabetes mellitus (OR 3.69, p = 0.024), were at a higher risk of developing MACE.

There is scarcity of data describing overall mortality and cardiac events subsequent to normal stress echocardiography. 
Table III. Univariate and multivariate predictors for combined cardiovascular events (including non-fatal myocardial infarction and coronary revascularisation).

\begin{tabular}{|c|c|c|c|c|c|c|}
\hline \multirow[t]{2}{*}{ Variable } & \multicolumn{2}{|c|}{ No. (\%) } & \multicolumn{2}{|c|}{ Univariate analysis } & \multicolumn{2}{|c|}{ Multivariate analysis } \\
\hline & No $(n=887)$ & Yes $(n=14)$ & OR $(95 \% \mathrm{Cl})$ & p-value & OR $(95 \% \mathrm{CI})$ & p-value \\
\hline \multicolumn{7}{|c|}{ Stress echocardiogram and exercise stress ECG } \\
\hline Group A (both negative; $n=671$ ) & $648(96.6)$ & $23(3.4)$ & 1.00 & & 1.00 & \\
\hline Group B (Echo+ and ECG-; $n=47)$ & $18(38.3)$ & $29(61.7)$ & $45.39(22.09-93.28)$ & $<0.001$ & $26.35(12.11-57.42)$ & $<0.001$ \\
\hline Group C (Echo- and ECG+; $\mathrm{n}=122)$ & $115(94.3)$ & $7(5.7)$ & $1.72(0.72-4.09)$ & 0.224 & $2.02(0.82-4.98)$ & 0.125 \\
\hline Group D (both positive; $\mathrm{n}=61$ ) & $27(44.3)$ & $34(55.7)$ & $35.48(18.44-68.26)$ & $<0.001$ & $19.84(9.60-40.78)$ & $<0.001$ \\
\hline \multicolumn{7}{|l|}{ Age group (yr) } \\
\hline$<50(\mathrm{n}=194)$ & $187(96.4)$ & $7(3.6)$ & 1.00 & & 1.00 & \\
\hline $50-59(n=245)$ & $213(86.9)$ & $32(13.1)$ & $4.01(1.73-9.31)$ & 0.001 & $2.39(0.83-6.90)$ & 0.109 \\
\hline $60-69(n=220)$ & $196(89.1)$ & 24 (10.9) & $3.27(1.38-7.78)$ & 0.007 & $1.40(0.46-4.27)$ & 0.552 \\
\hline $70-79(n=179)$ & $160(89.4)$ & 19 (10.6) & $3.17(1.30-7.74)$ & 0.011 & $1.24(0.39-3.90)$ & 0.715 \\
\hline$\geq 80(n=63)$ & $52(82.5)$ & $11(17.5)$ & $5.65(2.09-15.3)$ & 0.001 & $1.55(0.42-5.75)$ & 0.512 \\
\hline \multicolumn{7}{|l|}{ Gender } \\
\hline Female $(n=355)$ & $330(93.0)$ & $25(7.0)$ & 1.00 & & 1.00 & \\
\hline Male $(n=546)$ & 478 (87.5) & $68(12.5)$ & $1.88(1.16-3.03)$ & 0.010 & $1.26(0.66-2.41)$ & 0.491 \\
\hline \multicolumn{7}{|l|}{ Ethnicity } \\
\hline Chinese $(n=642)$ & $581(90.5)$ & $61(9.5)$ & 1.00 & & 1.00 & \\
\hline Malay $(n=159)$ & $132(83.0)$ & $27(17.0)$ & $1.95(1.19-3.18)$ & 0.008 & $1.47(0.74-2.90)$ & 0.268 \\
\hline Other $(n=100)$ & $95(95.0)$ & $5(5.0)$ & $0.50(0.20-1.28)$ & 0.149 & $0.48(0.16-1.47)$ & 0.199 \\
\hline \multicolumn{7}{|l|}{ No. of comorbidities* } \\
\hline $0(n=281)$ & $276(98.2)$ & $5(1.8)$ & 1.00 & & 1.00 & \\
\hline $1(n=180)$ & $167(92.8)$ & $13(7.2)$ & $4.30(1.51-12.3)$ & 0.006 & $2.08(0.61-7.02)$ & 0.240 \\
\hline$>1(n=440)$ & $365(83.0)$ & $75(17.0)$ & $11.3(4.53-28.4)$ & $<0.001$ & $3.69(1.19-11.5)$ & 0.024 \\
\hline \multicolumn{7}{|l|}{ Previous history of IHD and/or CABG } \\
\hline No $(n=693)$ & $655(94.5)$ & $38(5.5)$ & 1.00 & & 1.00 & \\
\hline Yes $(n=208)$ & $153(73.6)$ & $55(26.4)$ & $6.20(3.95-9.71)$ & $<0.001$ & $1.65(0.85-3.22)$ & 0.141 \\
\hline \multicolumn{7}{|l|}{ Clopidogrel } \\
\hline No $(n=800)$ & 735 (91.9) & $65(8.1)$ & 1.00 & & 1.00 & \\
\hline Yes $(n=100)$ & $72(72.0)$ & $28(28.0)$ & $4.40(2.65-7.29)$ & $<0.001$ & $1.51(0.73-3.11)$ & 0.269 \\
\hline \multicolumn{7}{|l|}{ Aspirin } \\
\hline No $(n=627)$ & $600(95.7)$ & $27(4.3)$ & 1.00 & & 1.00 & \\
\hline Yes $(n=273)$ & $207(75.8)$ & $66(24.2)$ & 7.09 (4.41-11.39) & $<0.001$ & $1.93(0.99-3.75)$ & 0.053 \\
\hline \multicolumn{7}{|l|}{ ACEI/ARB and/or $\beta$-blocker } \\
\hline No $(n=462)$ & $444(96.1)$ & $18(3.9)$ & 1.00 & & 1.00 & \\
\hline Yes $(n=438)$ & $363(82.9)$ & $75(17.1)$ & $5.10(2.99-8.68)$ & $<0.001$ & $1.08(0.50-2.32)$ & 0.848 \\
\hline
\end{tabular}

$\mathrm{OR}=1.00$ was reference category. Dashes (-) indicate OR was not calculable due to small frequency. ${ }^{*}$ Included hypertension, hyperlipidaemia and diabetes mellitus. ACEl: angiotensin-converting enzyme inhibitor; ARB: angiotensin II receptor blocker; CABG: coronary artery bypass grafting; Cl: confidence interval; ECG: electrocardiogram; ECG-: negative stress electrocardiogram; ECG+: positive stress electrocardiogram; Echo-: negative stress echocardiography; Echo+: positive stress echocardiography; IHD: ischaemic heart disease; OR: odds ratio

McCully et al ${ }^{(13)}$ reported a favourable outcome in 1,325 patients with normal exercise echocardiography. During a mean follow-up of 23 months, all cause-mortality, non-fatal myocardial infarction and coronary revascularisation were reported in 17 and 20 patients, respectively. Survival rates free of all cardiac events (e.g. cardiac death, non-fatal myocardial infarction and coronary revascularisation) at one year and three years were $99.5 \%$ and $98.6 \%$, respectively.

For our study, we found that the 18-month negative predictive value for MACE in patients with normal stress echocardiography was high and unaffected by the type of stress ECG response
(95.8\% and $92.6 \%$ for patients with negative stress ECGs and positive stress ECGs, respectively). Kobal et al ${ }^{(3)}$ and Mahenthiran et $\mathrm{al}^{(4)}$ had similarly demonstrated in their previous studies that a normal wall motion response, even in the setting of an ischaemic (positive) stress ECG response, portends a benign prognosis for patients undergoing stress echocardiography.

In our study, there was a $15.4 \%$ incidence of positive stress ECG response among patients with normal stress echocardiography findings. This was not unexpected given the fact that the reported specificity of the exercise electrocardiographic treadmill test (i.e. stress ECG) for 
Table IV. Univariate and multivariate predictors for mortality.

\begin{tabular}{|c|c|c|c|c|c|c|}
\hline \multirow[t]{2}{*}{ Variable } & \multicolumn{2}{|c|}{ No. (\%) } & \multicolumn{2}{|c|}{ Univariate analysis } & \multicolumn{2}{|c|}{ Multivariate analysis } \\
\hline & No $(n=887)$ & Yes $(n=14)$ & OR $(95 \% \mathrm{Cl})$ & p-value & OR $(95 \% \mathrm{Cl})$ & p-value \\
\hline \multicolumn{7}{|c|}{ Stress echocardiogram and exercise stress ECG } \\
\hline Group A (both negative; $\mathrm{n}=671$ ) & $665(99.1)$ & $6(0.9)$ & 1.00 & & 1.00 & \\
\hline Group B (Echo+ and ECG-;n = 47) & $46(97.9)$ & $1(2.1)$ & $2.41(0.28-20.4)$ & 0.420 & $0.99(0.11-9.07)$ & 0.994 \\
\hline Group C (Echo- and ECG $+\mathrm{n}=122)$ & $120(98.4)$ & $2(1.6)$ & $1.85(0.37-9.26)$ & 0.456 & $2.36(0.43-12.91)$ & 0.322 \\
\hline Group D (both positive; $\mathrm{n}=61$ ) & $56(91.8)$ & $5(8.2)$ & $9.90(2.93-33.4)$ & $<0.001$ & $4.54(1.18-17.50)$ & 0.028 \\
\hline \multicolumn{7}{|l|}{ Age group (yr) } \\
\hline$<50(n=194)$ & $192(99.0)$ & $2(1.0)$ & 1.00 & & 1.00 & \\
\hline $50-59(n=245)$ & $243(99.2)$ & $2(0.8)$ & $0.79(0.11-5.67)$ & 0.815 & $0.28(0.03-2.29)$ & 0.233 \\
\hline $60-69(n=220)$ & $216(98.2)$ & $4(1.8)$ & $1.78(0.32-9.81)$ & 0.509 & $0.76(0.11-5.36)$ & 0.784 \\
\hline $70-79(n=179)$ & $175(97.8)$ & $4(2.2)$ & $2.19(0.40-12.1)$ & 0.368 & $0.96(0.14-6.80)$ & 0.964 \\
\hline$\geq 80(n=63)$ & $61(96.8)$ & $2(3.2)$ & $3.15(0.43-22.8)$ & 0.257 & $1.07(0.12-9.89)$ & 0.955 \\
\hline \multicolumn{7}{|l|}{ Gender } \\
\hline Female $(n=355)$ & $352(99.2)$ & $3(0.8)$ & 1.00 & & 1.00 & \\
\hline Male $(n=546)$ & $535(98.0)$ & $11(2.0)$ & $2.41(0.67-8.71)$ & 0.179 & $1.70(0.40-7.18)$ & 0.472 \\
\hline \multicolumn{7}{|l|}{ Ethnicity } \\
\hline Chinese $(n=642)$ & $632(98.4)$ & $10(1.6)$ & 1.00 & & 1.00 & \\
\hline Malay $(n=159)$ & $155(97.5)$ & $4(2.5)$ & $1.63(0.51-5.27)$ & 0.414 & $1.31(0.37-4.63)$ & 0.680 \\
\hline Other $(n=100)$ & $100(100)$ & $0(0.0)$ & - & 0.997 & - & 0.996 \\
\hline \multicolumn{7}{|l|}{ No. of comorbidities* } \\
\hline $0(n=281)$ & $279(99.3)$ & $2(0.7)$ & 1.00 & & 1.00 & \\
\hline $1(n=180)$ & $179(99.4)$ & $1(0.6)$ & $0.78(0.07-8.66)$ & 0.839 & $0.13(0.01-1.78)$ & 0.126 \\
\hline$>1(n=440)$ & $429(97.5)$ & $11(2.5)$ & $3.58(0.79-16.34)$ & 0.099 & $0.29(0.05-1.87)$ & 0.193 \\
\hline \multicolumn{7}{|l|}{ Previous history of IHD and/or CABG } \\
\hline No & $688(99.3)$ & $5(0.7)$ & 1.00 & & 1.00 & \\
\hline Yes & $199(95.7)$ & $9(4.3)$ & $6.22(2.06-18.8)$ & 0.001 & $1.82(0.50-6.72)$ & 0.366 \\
\hline \multicolumn{7}{|l|}{ Clopidogrel } \\
\hline No & $789(98.6)$ & $11(1.4)$ & 1.00 & & 1.00 & \\
\hline Yes & $97(97.0)$ & $3(3.0)$ & $2.22(0.61-8.09)$ & 0.227 & $0.71(0.17-2.93)$ & 0.630 \\
\hline \multicolumn{7}{|l|}{ Aspirin } \\
\hline No $(n=627)$ & $624(99.5)$ & $3(0.5)$ & 1.00 & & 1.00 & \\
\hline Yes $(n=273)$ & $262(96.0)$ & $11(4.0)$ & $8.73(2.42-31.6)$ & 0.001 & $2.04(0.48-8.60)$ & 0.331 \\
\hline ACEI/ARB and/or $\beta$-blocker & $624(99.5)$ & $3(0.5)$ & 1.00 & & 1.00 & \\
\hline No $(n=462)$ & $462(100.0)$ & 0 & 1.00 & & 1.00 & \\
\hline Yes $(n=438)$ & $424(96.8)$ & $14(3.2)$ & - & 0.992 & - & 0.992 \\
\hline
\end{tabular}

$\mathrm{OR}=1.00$ was reference category. Dashes (-) indicate OR was not calculable due to small frequency. *Included hypertension, hyperlipidaemia and diabetes mellitus. ACEl: angiotensin-converting enzyme inhibitor; ARB: angiotensin II receptor blocker; CABG: coronary artery bypass grafting; Cl: confidence interval; ECG: electrocardiogram; ECG-: negative stress electrocardiogram;ECG+: positive stress electrocardiogram; Echo-: negative stress echocardiography; Echo+: positive stress echocardiography; IHD: ischaemic heart disease; OR: odds ratio

diagnosing ischaemic heart disease was $77 \% .{ }^{(14,15)}$ Managing cardiologists, not uncommonly, have to manage patients with negative stress echocardiography but positive (discordant) stress ECG findings. Our results will help to reassure cardiologists that such results do not portend a higher risk of MACE when compared to those of patients with negative stress echocardiography and negative (concordant) stress ECG.

In our study, $4.9 \%$ of patients in Group C (negative stress echocardiography but positive stress ECG) had diagnostic coronary angiography at the managing physicians' discretion and subsequently $\mathrm{PCl}$. The 2007 American Society of Echocardiography document on stress echocardiography reported an average sensitivity of $88 \%(1,265 / 1,445$ patients) and average specificity of $83 \%$ (465/563 patients) for stress echocardiography for the detection of coronary artery stenosis (generally over $50 \%$ diameter stenosis by angiography), based on data pooled from available studies. ${ }^{(5)}$ Due to the inherent specificity of $83 \%$ for stress echocardiography, managing physicians should pay due consideration to the pretest probability and consider further diagnostic tools if the index of suspicion for coronary artery stenosis is high despite the stress echocardiography being negative.

For stress echocardiography, patients with positive stress echocardiography will have a higher risk of developing MACE. 
On the other hand, our study demonstrated that discordant results (negative stress echocardiogram but positive stress ECG) in patients do not portend a higher risk of MACE as compared to concordant results (both stress echocardiogram and stress ECG are negative). However, further investigation may be warranted if the index of suspicion for coronary artery stenosis is high despite negative stress echocardiography.

This study was not without limitations. This was a retrospective cohort study that followed up patients for 18 months from the test date. Patients in our database are still being followed for analysis of results over a longer period of time. The study was conducted at a single centre. However, the patient population attended to by $\mathrm{CGH}$ is representative of the ethnic make-up of Singapore and Southeast Asia, in general. Stress echocardiography images were all captured and interpreted by various echocardiologists who were on duty, and this could have resulted in inter-rater variability. However, this could possibly be viewed positively, as it replicates real-life situations wherein stress echocardiography is generally performed by echocardiologists on duty at healthcare centres instead of single operators at a core echocardiography laboratory.

\section{ACKNOWLEDGEMENTS}

The authors thank the Clinical Measurement Unit at $\mathrm{CGH}$, Singapore, for its unwavering support on echocardiography.

\section{REFERENCES}

1. Krivokapich J, Child JS, Gerber RS, Lem V, Moser D. Prognostic usefulness of positive or negative exercise stress echocardiography for predicting coronary events in ensuing twelve months. Am J Cardiol 1993; 71:646-5.

2. Marwick TH, Shaw L, Case C, Vasey C, Thomas JD. Clinical and economic impact of exercise electrocardiography and exercise echocardiography in clinical practice. Eur Heart J 2003; 24:1153-63.

3. Kobal SL, Wilkof-Segev R, Patchett MS, et al. Prognostic value to myocardial ischemic electrocardiographic response in patients with normal stress echocardiographic study. Am J Cardiol 2014; 113:945-9.

4. Mahenthiran J, Bangalore S, Yao SS, Chaudhry FA. Comparison of prognostic value of stress echocardiography versus stress electrocardiography in patients with suspected coronary artery disease. Am J Cardiol 2005; 96:628-34.

5. Pellikka PA, Nagueh SF, Elhendy AA, Kuehl CA, Sawada SG; American Society of Echocardiography. American Society of Echocardiography recommendations for performance, interpretation and application of stress echocardiography. J Am Soc Echocardiogr 2007; 20:1021-41.

6. Zacharias K, Ahmadvazir S, Ahmed A, et al. Relative diagnostic, prognostic and economic value of stress echocardiography versus exercise electrocardiography as initial investigation for the detection of coronary artery disease in patients with new onset suspected angina. Int J Cardiol Heart Vasc 2015; 7:124-30.

7. Zacharias K, Ahmed A, Shah BN, et al. Relative clinical and economic impact of exercise echocardiography vs. exercise electrocardiography, as first line investigation in patients without known coronary artery disease and new stable angina: a randomized prospective study. Eur Heart J Cardiovasc Imaging 2017; 18:195-202.

8. Peteiro J, Monserrat L, Vazquez E, et al. Comparison of exercise echocardiography to exercise electrocardiographic testing added to echocardiography at rest for risk stratification after uncomplicated acute myocardial infarction. Am J Cardiol 2003; 92:373-6.

9. Severi S, Picano E, Michelassi C, et al. Diagnostic and prognostic value of dipyridamole echocardiography in patients with suspected coronary artery disease. Comparison with exercise electrocardiography. Circulation 1994; 89:1160-73.

10. Sicari R, Nihoyannopoulos P, Evangelista A, et al. Stress echocardiography expert consensus statement--executive summary: European Association of Echocardiography (EAE) (a registered branch of the ESC). Eur Heart J 2009; 30:278-89.

11. Bigi R, Desideri A, Galati A, et al. Incremental prognostic value of stress echocardiography as an adjunct to exercise electrocardiography after uncomplicated myocardial infarction. Heart 2001; 85:417-23.

12. Steinberg EH, Madmon L, Patel CP, et al. Long-term prognostic significance of dobutamine echocardiography in patients with suspected coronary artery disease: results of a 5-year follow-up study. J Am Coll Cardiol 1997; 29:969-73.

13. McCully RB, Roger VL, Mahoney DW, et al. Outcome after normal exercise echocardiography and predictors of subsequent cardiac events: follow-up of 1,325 patients. J Am Coll Cardiol 1998; 31:144-9.

14. Chaowalit N, McCully RB, Callahan MJ, et al. Outcome after normal dobutamine stress echocardiography and predictors of adverse events: long-term follow-up of 3014 patients. Eur Heart J 2006; 27:3039-44.

15. Detrano R, Gianrossi R, Froelicher V. The diagnostic accuracy of the exercise electrocardiogram: a meta-analysis of 22 years of research. Prog Cardiovasc Dis 1989; 32:173-206. 